\section{Product Line Architecture for Automatic Evolution of Multi-Tenant Applications}

\author{
View Document
}

3

Author(s)

\section{Related Articles}

\begin{tabular}{l|l|l} 
Virtual & Integral & A statistical \\
Machine & Federated & based \\
Based Energy- & Identity & resource \\
Efficient Data & Management & allocation \\
Cente... & for Cloud ... & scheme in...
\end{tabular}

Jose-Miguel Horcas ; $\quad$ Monica Pinto ; Lidia Fuentes

Abstract

Authors

Figures

References

Citations

Keywords

Metrics

Media

\begin{abstract}
:
Cloud computing is becoming the predominant mechanism to seamlessly deploy applications with special requirements such as massive storage sharing or load balancing, usually provided as services by cloud platforms. A developer can improve the application's delivery and productivity by following a multi tenancy approach, where variants of the same application can be quickly customized to the necessities of each tenant. However, managing the inherent variability existing in multi-tenant applications and, even more importantly, managing the evolution of a multi-tenant application with hundreds of tenants and thousands of different valid architectural configurations can become intractable if performed manually. In this paper we propose a product line architecture approach in which: (1) we use cardinality-based variability models to model each tenant as a clonable feature, (2) we automate the process of evolving the multi- tenant application architecture, and (3) we demonstrate that the implemented process is correct and efficient for a high number of tenants in a reasonable time. We use a running case study in the domain of medical software.
\end{abstract}

Published in: Enterprise Distributed Object Computing Conference (EDOC), 2016 IEEE 20th International

Date of Conference: 5-9 Sept. 2016

INSPEC Accession Number: 16342244

Date Added to IEEE Xplore: 29 September 2016

DOI: 10.1109/EDOC.2016.7579384

ISBN Information:

Publisher: IEEE

Electronic ISBN: 978-1-4673-9885-5

Print on Demand(PoD) ISBN: 978-1-4673-9886-2

Electronic ISSN: 2325-6362

Download PDF
Download Citations
View References
Email
Print

Request Permissions

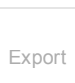

Export

Share

Alerts
Read the full document

Abstract

\section{Keywords}

\section{IEEE Keywords}

Hospitals, Cloud computing, Computer architecture, Authentication, Virtual machining, Surgery

Authors

Figures

INSPEC: Controlled Indexing

References

software product lines, cloud computing, software architecture

Citations

INSPEC: Non-Controlled Indexing

product line architecture, medical software, multitenant application architecture, clonable feature,

Keywords cardinality-based variability models, automatic evolution, architectural configurations, cloud computing
Back to Top

\title{
Authors
}

Jose-Miguel Horcas

Andalucia Tech, Univ. de Malaga, Malaga, Spain

Related Articles 
» Virtual Machine Based EnergyEfficient Data

Cente...

Kejiang Ye; Dawei Huang; ...

» Integral Federated Identity

Management for

Cloud ..

Maicon Stihler;

Altair Ol...

" A statistical based resource allocation scheme in..

Zhenzhong Zhang;

Haiyan W..

" Infrastructure

Deployment Over

the Cloud

Carlos de Alfonso;

Miguel...

(securing user authentication using single sign-

on...

Ashish G. Revar; Madhuri ..

» Optimal Virtual Machine

Placement in

Large-Scale ...

Hana Teyeb; Ali

Balma; Ne...

" Design and

Deployment of a

Trusted

Eucalyptus Clo..

Imran Khan; Habib-

ur Rehm...

" An Agent Based

Architecture for

VM Software

Track...

Fabrizio Messina;

Giusepp...

» Building a

Distributed Block

Storage System

for $\mathrm{C}$...

Xiaoming Gao; Yu

Ma; Marl...

" Scalable and

elastic e-

Assessment cloud

solution

Sasko Ristov;

Marjan Guse...
Andalucia Tech, Univ. de Malaga, Malaga, Spain

Lidia Fuentes

Andalucia Tech, Univ. de Malaga, Malaga, Spain

\section{IEEE Account}

»Change Username/Password

" Update Address
Purchase Details

»Payment Options

» Order History

»View Purchased Documents
Profile Information

»Communications Preferences

»Profession and Education

» Technical Interests
Need Help?

" US \& Canada: +1 8006784333

"Worldwide: +1 7329810060

»Contact \& Support 
About IEEE Xplore | Contact Us | Help | Terms of Use | Nondiscrimination Policy | Sitemap | Privacy \& Opting Out of Cookies

A not-for-profit organization, IEEE is the world's largest technical professional organization dedicated to advancing technology for the benefit of humanity. C Copyright 2016 IEEE - All rights reserved. Use of this web site signifies your agreement to the terms and conditions. 